\title{
Desarrollo convergente municipal entre estados contiguos a Nayarit y Sinaloa
}

\section{Convergent municipal development between contiguous states to Nayarit and Sinaloa}

\author{
Eduardo Meza-Ramos \\ Zulema Berenice Naya-Flores*
}

\begin{abstract}
The analysis of the economical growth shows unlike sectors in the countries, among the rural and the urban areas, and among the wealthy and the low-developed areas, too; in México, the policy of trade liberalization has not been reflected, in general, in the wealth of the Mexican population. In this paper, the hypothesis of convergence at a county level of the Mexican states of Chihuahua, Durango, Jalisco, Nayarit, Sinaloa, Sonora y Zacatecas was evaluated; with the data considered in the study we can argue the existence of $\sigma$ and $\beta$ convergence, given, in average, a Standard Deviation of 1.73 in 1989, and this indicator came down to 1.31 in 2006. The $\beta$ convergence shows a negative relationship with an absolute value of its statistical bigger than 2; at 95\% of confidence. Even though there are social and sector policies, there is a notorious lack of public policies that promote the local development.
\end{abstract}

Keywords: converging development of counties, per capita gross national product.

\begin{abstract}
Resumen
El análisis del crecimiento económico registra disparidades sectoriales que se manifiestan en el interior de los países, entre las áreas urbanas y las rurales; entre las regiones prósperas y las rezagadas. En México la política de liberalización comercial no se ha visto reflejada de manera generalizada en la riqueza de la población. Se evaluó la hipótesis de convergencia en el ámbito municipal de los estados de Chihuahua, Durango, Jalisco, Nayarit, Sinaloa, Sonora y Zacatecas; por los datos considerados en el estudio se puede argumentar la existencia de convergencia $\sigma$ y $\beta$, pues en promedio, la desviación estándar en el año 1989 fue de 1.73 y disminuyó a 1.31 en 2006 . La convergencia $\beta$ describe una relación negativa con un valor absoluto de su estadístico mayor a 2; con $95 \%$ de confianza. Cabe señalar que se cuenta con políticas sociales y sectoriales pero se carece de políticas públicas que promuevan el desarrollo regional.
\end{abstract}

Palabras clave: desarrollo convergente municipal, producto interno bruto per cápita.

* Universidad Autónoma de Sinaloa, México. Correos-e: eduarmezara33@hotmail.com, naya_flores@hotmail.com. 


\section{Introducción}

Este estudio de economía regional busca explicar cuáles son los factores económicos e institucionales que influyen en la convergencia o divergencia de las tasas de crecimiento económico, propone respuestas a la pregunta de ¿por qué algunas regiones, con el transcurso del tiempo, se vuelven ricas y otras pobres? Cuestión que es básica para explicar el desarrollo de los pueblos en periodos determinados.

El análisis corresponde al tiempo en que México comenzó la apertura comercial y su incorporación a los organismos internacionales. Proceso que transitó por las tres etapas siguientes: 1) la asociada con el ingreso al Acuerdo General sobre Aranceles Aduaneros y Comercio (GATT) en 1985, que se caracterizó por una reducción unilateral de los aranceles sobre los bienes intermedios y de capital; 2) en el año 1988 se extendió la estrategia anterior a la mayoría de los bienes de consumo, lo que disminuyó significativamente la dispersión arancelaría y las restricciones cuantitativas a las importaciones, y 3) la que se ha tipificado como generadora de expectativas de toda índole que se inauguró en enero de 1994 con la entrada en vigor del Tratado de Libre Comercio de América del Norte (TLCAN) (Ocegueda, 2007), lo que redujo de forma drástica los aranceles, pero vino acompañada del despertar indígena de México, al alzarse simbólicamente un grupo de gente autóctona de Chiapas.

A fin de comprender y mostrar la tendencia del crecimiento de los estados que rodean a Nayarit y Sinaloa, este trabajo analiza las disparidades económicas mediante la prueba de convergencia- $\sigma$ y convergencia- $\beta$, utilizando un modelo econométrico de mínimos cuadrados no lineales. En términos generales, el estudio de la zona se analiza a partir del producto interno bruto per cápita (PIBPC) en 397 municipios, de los cuales en el estado de Sinaloa se ubican 18, Nayarit aporta 20, Durango 39, Zacatecas 57, el estado grande de México, Chihuahua, está formado por 67, Sonora por 72 y Jalisco es el estado que reporta mayor número de comunas, pues está dividido en 126.

A su vez, se calculó un modelo econométrico de corte transversal para el periodo 1989-2006, para evaluar la hipótesis de convergencia. Cabe señalar que la regionalización oficial ubica a Nayarit en la región centrooccidente de México, por lo que se sabe poco de la relación que se tiene con Sinaloa, que se le ubica en la región norte con Baja California, Baja California Sur, Chihuahua, Coahuila, Nuevo León, Sinaloa, Sonora y Tamaulipas. 
Precisamente para evaluar la convergencia en los estados contiguos a Nayarit y Sinaloa, ${ }^{1}$ de los cuales Jalisco está caracterizado como entidad industrial, Zacatecas con la tradición de desarrollo minero, Durango que busca su ingreso al desarrollo a partir de alianzas internacionales con Texas, Sinaloa que se destaca por la agroindustria, y los estados fronterizos de Sonora y Chihuahua que al parecer han sido favorecidos por el TLCAN, así como por su situación geográfica de vecindad con Estados Unidos y que reportan cierto desarrollo en la zona fronteriza. En esas entidades, a partir del ingreso de la población, en el ámbito administrativo municipal, se mide la convergencia y su velocidad, no obstante que en la situación actual del capitalismo mexicano, las entidades describen diferencias en el nivel de desarrollo y han tenido diferentes efectos por la apertura comercial.

En lo referente a las limitaciones de este trabajo, cabe señalar que se utilizó información municipal de 1989, 2004 y 2006, ya que los coeficientes técnicos del VAB del año 2004 se utilizaron para asignar el PIB municipal de 2006. Por observancia del artículo 38 de la Ley de Información Estadística y Geográfica, en algunos casos el INEGI no informa los datos de algunas actividades que pudieran vulnerar el principio de confidencialidad, sin embargo, con la información disponible se calculó el índice de PIBPC municipal, y se omitieron algunos municipios que en el primer ańo no reportaban actividades económicas, informaban VAB negativo, así como a los municipios de más reciente creación, que no consignan información.

Por otro lado, con este estudio se contribuye al debate del estado de bienestar social de la población que vive en los municipios de México, considerando el ingreso per cápita como indicador de desarrollo social que permite caracterizar la riqueza de los habitantes de una región y compararla con su entorno, para el caso de los municipios en los estados que colindan con Sinaloa y Nayarit, no obstante el desarrollo desigual y el tamaño de las economías.

Con este trabajo se aportan indicadores municipales para contribuir a resolver la ausencia de datos relativos a la intensidad de la interacción económica entre municipios, tan necesaria por la creciente apertura comercial y la intensa reestructuración productiva instrumentada últimamente, que contribuyan a relacionar la actividad económica espacial, es decir, con el entorno geográfico regional.

El trabajo comprende cinco apartados, el primero describe los fundamentos teóricos, básicamente la convergencia tipo $\sigma$ y la convergencia

\footnotetext{
${ }^{1}$ La región de estudio se localiza al norte de México, colindando con los estados de Arizona, Nuevo México y Texas de Estados Unidos, y hasta al sur con el golfo de California y el océano Pacífico; al este con Baja California y al oeste con Coahuila, San Luis Potosí, Guanajuato, Michoacán y Colima.
} 
tipo $\beta$, señalando que antes de los años ochenta se fundamentó empíricamente la convergencia entre los estados de la República, pero a partir de esa fecha ha cobrado importancia la divergencia. En el segundo se describe la metodología, que partió de reunir información de los censos económicos y de población. En el tercero se construyeron las variables que generalmente se utilizan para medir la convergencia, en este caso el PIB y su tasa de crecimiento. También se presenta el modelo econométrico que se utilizó para fundamentar empíricamente la tendencia de la convergencia, y con los datos de las variables se construyeron mapas temáticos de los municipios. En el apartado siguiente se presenta el cómputo de la hipótesis de convergencia, posteriormente se abordan los resultados del modelo econométrico, destacando que el valor de convergencia $\sigma$ es decreciente en el periodo y el estadístico de convergencia $\beta$ es negativo y significativo en el ámbito de las regiones y de cada uno de los estados. Finalmente se exponen las conclusiones, donde se destacan los hallazgos más relevantes de la investigación.

\section{Fundamentos teóricos}

El crecimiento económico registra disparidades sectoriales que se manifiestan en el interior de los países, entre las áreas urbanas y las rurales; entre las regiones prósperas y las rezagadas; entre las áreas metropolitanas y las ciudades medianas y pequeñas, situación que se analiza a partir de la hipótesis de convergencia esbozada por Solow (1956), que preveía que a largo plazo la tasa de crecimiento en el ingreso per cápita tiende a disminuir, a medida que aumenta el nivel de ingreso per cápita. Por otra parte Sala-i-Martin (1994) propone el análisis a partir de la distribución del ingreso per cápita, medida por la desviación estándar del ingreso por habitante durante un periodo a partir de convergencia $\sigma$ (sigma) y convergencia $\beta$ (beta).

En México se ha documentado ampliamente el proceso de convergencia absoluta o relativa entre los estados, durante el periodo previo a la liberación económica (Esquivel, 1999), pero a partir de la liberación comercial ha cobrado relevancia la hipótesis de que el país ha experimentado un proceso de divergencia regional, por lo que muchos estudios toman como región a un estado y como componentes a los municipios para enfocar el análisis de crecimiento regional. Valdivia (2007: 2) presenta una perspectiva de convergencia condicional donde se reconoce que las regiones convergen a su propio estado estacionario, con lo cual se identifican las diferencias regionales. En tanto que las escuelas de pensamiento tradicionales, como la clásica, neoclásica y keynesiana, conceden especial importancia a la movilidad de factores (capital y trabajo), han 
sido la piedra angular mediante la cual se ha generado el desarrollo teórico y el diseño de políticas regionales en las últimas décadas en todo el mundo.

El deterioro del bienestar social y la polarización económica regional suele atribuirse a las fallas y limitaciones de la estrategia de desarrollo, es probable que esa evaluación sea injusta al dejar de lado el efecto que han tenido los choques económicos externos, los errores en el diseño de políticas públicas compensatorias y las fallas en el manejo de la política macroeconómica que ha restado competitividad a la planta productiva, sesgando la especialización nacional hacia las exportaciones de bienes maquilados y hacia la producción de bienes no comerciables (Ros y Skott, 1995).

Como se ha documentado, después de la apertura comercial, la economía mexicana ha experimentado un proceso de divergencia regional, por lo que el análisis del crecimiento regional se ha orientado hacia una perspectiva condicional abonada por el capital humano (Barceinas y Raymond, 2005), de apertura comercial (Díaz-Bautista y Díaz, 2003) o por la inversión pública (Rodríguez-Orregia, 2005), pero esos estudios siguen una línea de investigación empírica en el ámbito estatal, que han encontrado convergencia regional en el país. En cuanto a los estados, Ocegueda (2007: 119) señala que Campeche, Chiapas, Guerrero, Nayarit, Oaxaca, Sinaloa, Tabasco, Veracruz y Zacatecas no han recibido beneficios tangibles de la apertura comercial, pero que los beneficios son más que evidentes en Baja California, Chihuahua, Coahuila, Colima, Distrito Federal, Nuevo León, Puebla, Querétaro y Yucatán. Por otra parte, Aguascalientes, Estado de México, Hidalgo, Jalisco, Michoacán, Sonora y Tamaulipas percibieron beneficios moderados; en tanto que las siete entidades restantes: Baja California Sur, Durango, Guanajuato, Morelos, Quintana Roo, San Luis Potosí y Tlaxcala describen una situación poco clara, al medir el efecto de la apertura económica sobre el crecimiento de las regiones y estados de la República.

Con la globalización se ha formado un conjunto de nodos, en especial de ciudades que responden a las exigencias del proceso de acumulación a escala mundial. Pero esto no supone eliminar el problema de las desigualdades regionales, aunque se observa una relativa reconfiguración del territorio, dada por una presencia más dispersa de las empresas en función de las ventajas que han ofrecido algunos gobiernos estatales que, ante la falta de claridad de destino de las políticas económicas nacionales y su direccionamiento regional, fincan sus expectativas de mejoramiento económico en las oportunidades de inversión y desarrollo que podría ofrecer el tLCAN y otros países fuera de esta área de influencia (Corona y Ángel, 2003). 
Otro estudio que considera el comportamiento registrado por el PIB per cápita en el periodo 1993-1999 (Messmacher, 2000), muestra un aumento en la desigualdad regional absoluta a partir de 1993, aunque la dispersión relativa no aumentó. Según este análisis, los cambios estructurales en la economía parecen haber tenido dos efectos: 1) en el sectorial, la tasa de crecimiento del sector manufacturero se incrementó sustancialmente a partir de 1993 y se expandió más que cualquier otro sector, a excepción del transporte, almacenaje y comunicaciones. Por este efecto crecieron a mayores tasas los estados donde la manufactura representa una elevada proporción de la producción; y 2) en lo regional, los estados del norte han tenido un mejor desempeño que el esperado, dada la estructura de su producción.

Desde el punto de vista teórico, comúnmente se afirma que un modelo económico da lugar a convergencia si las variables más relevantes alcanzan un nivel de equilibrio a largo plazo de una forma determinada; más concretamente, la tasa de crecimiento debe tender a su valor de estado estacionario siguiendo una trayectoria monotónicamente decreciente (Villaverde y Sánchez, 1998: 178). En tanto que la arquitectura de la economía global se fundamenta en una red interactiva que forma un sistema de varios niveles interrelacionados, para dar respuesta a los crecientes imperativos de una economía más globalizada que requiere desarrollar potencialidades locales y regionales, así como sistemas de cooperación en las localizaciones intrarregionales.

En la literatura sobre convergencia, las regiones se consideran entes independientes de su localización espacial y de sus conexiones con otras regiones (Krugman, 1991; Quah, 1999, citados en Aroca y Bosch, 2000: 200-201. Estos autores señalan que no obstante que desde el punto de vista teórico son abundantes las aportaciones para apoyar la idea de que la geografía puede ser un determinante de los procesos de desarrollo regional, esos estudios argumentan que bajo determinadas circunstancias pueden existir concentraciones espaciales de producción.

Los trabajos de Myrdal y Perroux contribuyeron para que la teoría del crecimiento económico transitara a las propuestas de la economía del desarrollo, al analizar las diferencias encontradas entre los diversos procesos de crecimiento desarrollados en el mundo (Aguado et al., 2009). Por otra parte, Meier y Seers (1986, citados por Aguado, 2009) señalan que la corriente de economistas formada por Rosenstein-Rodan, Nurkse, Singer, Lewis, Gerschenkron, Myrdal, Hirschman, Scitovsky, Perroux, Rostow, Myint y Prebisch, reconocidos como pioneros del desarrollo, proponían la intervención del Estado para movilizar los recursos ociosos.

Al estudiar la economía regional en México, Valdivia (2007: 13) realizó un estudio de convergencia para identificar los efectos de economía 
de escala, los efectos espaciales y la heterogeneidad estructural, considerando todos los municipios del país, y documentó que la dinámica de desigualdad regional que ha vivido la población durante los últimos 20 años, es posible que se deba a la fuerte presencia de efectos espaciales que además no son homogéneos a lo largo de las regiones sustantivas.

En ese trabajo se propuso una regionalización que se denominó centroperiferia a partir de la autocorrelación espacial de la productividad en el ámbito municipal, la región central se formó con municipios que en el año 2003 mostraron productividad del trabajo positiva, mientras que la región periferia reportaba una productividad negativa. Se especifica que su principal hallazgo fue mostrar los efectos espaciales, que es muy probable que estén operando en el proceso de convergencia regional en el país, pero que esos efectos espaciales difieren entre los municipios pobres $\mathrm{y}$ los ricos.

\section{Metodología}

Para fundamentar empíricamente este trabajo, se utilizó un modelo econométrico no lineal para explicar el desarrollo de la región de estudio, de acuerdo con la teoría de convergencia. Los municipios que se analizaron corresponden a los estados del norte y centro-occidente de México que colindan con Nayarit y Sinaloa. Las variables que se consideran en este modelo se construyeron de la siguiente manera: para obtener el PIBPC de cada municipio se utilizó información divulgada por el INEGI y otras estadísticas sobre población y actividades económicas, así como la serie del рів de los estados del país que elaboró Germán-Soto (2005). Los datos provienen de los censos económicos de los años 1989 y 2004 referentes al VAB que por las actividades de los sectores primario, secundario y terciario se generó en los municipios de Chihuahua, Durango, Jalisco, Nayarit, Sinaloa, Sonora y Zacatecas.

Por otra parte, para tener series afines a precios de 1993, primero se obtuvo el importe monetario del valor agregado censal bruto, correspondiente a cada municipio, luego se calculó el porcentaje correspondiente, a continuación se utilizó el importe del piв estatal reconocido oficialmente y se repartió de acuerdo con el porcentaje de vaB de cada municipio, mediante una regla de tres, para así tener el importe del pıв municipal.

En lo que se refiere al otro elemento del PIBPC, la población, a continuación se utilizó la información de los censos de población y vivienda de los años 1980, 1990 y 2000 y del conteo de población y vivienda de 2005; para obtener la población en el año que también corresponde al censo económico se calculó la tasa de crecimiento exponencial de la po- 
blación y se utilizó la cantidad correspondiente al ańo censal, el procedimiento se resume en la siguiente ecuación:

$$
P I B_{i, m}=P I B_{e} \mathrm{x} \% V A B_{i, m}
$$

Donde $P I B_{i, m}$ es el producto interno bruto de cada uno de los municipios; PIB es el importe estatal del producto interno bruto, reconocido por fuentes oficiales, y $\% V A B_{i, m}$ es el porcentaje del valor agregado bruto de cada uno de los municipios.

Por otra parte, para obtener el crecimiento de la población y el del PIBPC se utilizó la tasa de crecimiento exponencial que se expresa de la siguiente forma: $\mathrm{P}_{1}=\mathrm{P}_{0}(1+\mathrm{r})^{\mathrm{t}}$, donde $\mathrm{P}_{1}$ es la población final, $\mathrm{y} \mathrm{P}_{0}$ es la población al inicio del periodo, respectivamente, $t$ es el tiempo transcurrido y $r$ el factor de crecimiento; cuando se necesitaron los indicadores del PIBPC, para los años de estudio, se ajustó la formula señalada para ese caso.

Se organizaron los municipios de los estados que colindan con Nayarit, se le denominó región Nayarit, y otra con los de Sinaloa, se le nombró región Sinaloa, para realizar comparaciones entre ambas. Por otra parte, se elaboraron mapas del PIBPC de la región general de estudio para ilustrar las diferencias entre los años 1989 y 2006.

\subsection{Cómputo de la hipótesis de convergencia}

El análisis se inició a partir del indicador de convergencia- $\sigma$, que mide la dispersión del ingreso por habitante entre municipios, con el estadístico referido a la desviación estándar del ingreso por habitante. Si la desviación estándar disminuye, de un periodo a otro, se dice que tiende a la convergencia, por el contrario, si ese indicador reporta incremento, se interpreta que existe tendencia a la divergencia. La convergencia- $\sigma$, Asuad y Quintana (2006: 8) la indican con la siguiente ecuación:

$$
\sigma_{t}=\sqrt{\frac{1}{T} \sum_{i=1}^{n}\left(\log Y_{i T}-\log Y_{T}\right)^{2}}
$$

Donde $i$ es la región; $Y_{i T}$ el ingreso por habitante; $Y_{T}$ es el ingreso por habitante año base y $T$ el número de observaciones. El análisis de convergencia- $\sigma$ se puede completar por el de convergencia $\beta$, que daría cuenta de convergencia absoluta.

El modelo econométrico para calcular la convergencia se estimó siguiendo la metodología que ofrece Gujarati (2005: 158-187) para la 
solución de un modelo de regresión exponencial del tipo siguiente:

$$
Y_{i}=\beta_{1} X_{i}^{\beta 2 e^{\prime \prime} i}
$$

el cual se puede expresar alternativamente como el modelo log-log siguiente:

$$
\operatorname{Ln} Y_{i}=\mathrm{h} \beta_{1}+\beta_{2} \mathrm{~h} X_{i}+u_{i}
$$

Otros utilizan la ecuación

$$
\operatorname{Ln}\left[\left(Y_{i, t+T} / Y_{i, t}\right) / T\right]=a-\left(1-\exp \left(-\beta^{*} t\right)\right) / T^{*} \log \left(Y_{i, t}\right)+u_{i t}
$$

Donde $Y_{\mathrm{i}, \mathrm{t}+\mathrm{T}} / Y_{\mathrm{i}, \mathrm{t}}$ es el vector que contiene los valores del logaritmo de la relación $Y_{\mathrm{i}, \mathrm{t}+\mathrm{T}} / Y_{\mathrm{i}, \mathrm{t}}$, que representa la tasa de crecimiento promedio del ingreso per cápita durante el periodo y log $Y_{\mathrm{i}, \mathrm{e}}$, el ingreso per cápita en el primer año. La constante $a$ y los coeficientes $\beta$ 's son estadísticos y $u$ es el término estocástico que recoge los errores de medición. La ecuación 2.5 parte del supuesto teórico de que las economías tienen condiciones estructurales similares, por lo que se espera que sus estados estacionarios difieran entre sí. Por otra parte, la confirmación de la convergencia- $\beta$ implica que el parámetro $\beta$ resulte negativo, y si además $\beta$ es estadísticamente significativo, se considera evidencia suficiente para concluir que existe convergencia condicional. En casos como ése, se concluye que las economías convergen hacia sus propios estados estacionarios, los cuales son diferentes debido a la influencia que tienen sobre éstas las variables incluidas en el vector $X_{\mathrm{i}}$. Además, a partir del coeficiente $\beta$ es posible calcular la velocidad de convergencia, la cual se obtiene a partir de la siguiente expresión:

$$
\lambda_{\lambda}=-\frac{h(1+\beta)}{T}
$$

Como medida de la bondad de ajuste se utilizó el coeficiente de determinación $r^{2}$.

\section{Resultados del modelo econométrico}

Se elaboró y organizó información del valor agregado censal bruto a nivel municipal, del PIB por estados y de la población municipal, para obtener el PIBPC municipal de los años 1989 y 2006 para analizar la tendencia durante 17 ańos, periodo de mediano plazo, adecuado para calibrar el crecimiento en el ámbito municipal. 
Primero se procedió a obtener el PIBPC por municipio de los estados del primer año de estudio, 1989, observando que los municipios se marcan con los mismos colores, lo cual significa que en todos los estados existe dispersión de ingresos y desigualdad, aun cuando sean estados con cierto desarrollo industrial, agrícola o de servicios, situación que persiste en el año 2006 (mapas i y II).

Por otra parte, se obtuvo la tasa de crecimiento promedio del ingreso PC entre 1989 y 2006 en función del logaritmo del PIBPC de 1989 para los 397 municipios de la región de Nayarit y Sinaloa para buscar evidencia de convergencia económica, advirtiendo que los modelos teóricos de crecimiento económico suelen predecir que habrá convergencia, pero en las tasas de crecimiento de la renta influyen muchos factores. Martínez (2001) plantea que entre los factores que parecen ser muy importantes en el comportamiento de divergencia o convergencia son: las decisiones políticas de los gobernantes, la estructura jurídica del país, el grado de educación de sus habitantes, el nivel tecnológico y el grado de desarrollo inicial o volumen de capital preexistente.

\section{Mapa $\mathrm{I}^{2}$ \\ PIBPC 1989, a precios de 1993}

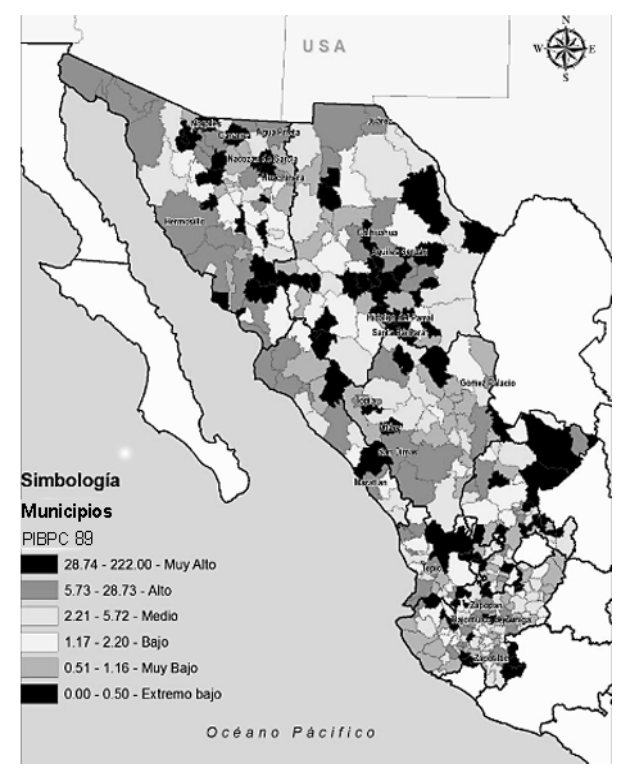

Fuente: Elaboración propia con datos de INEGI, SIMBAD Censos Económicos, 1989.

${ }^{2}$ Para la elaboración de los mapas se contó con el apoyo del profesor Pedro Luna Mayorquín y de A. Issai Jiménez Ante, en el diplomado de Iris, ofrecido en la Unidad Académica de Economía de la UAN, verano 2008. 


\section{Mapa II}

PIBPC 2006, a precios de 1993

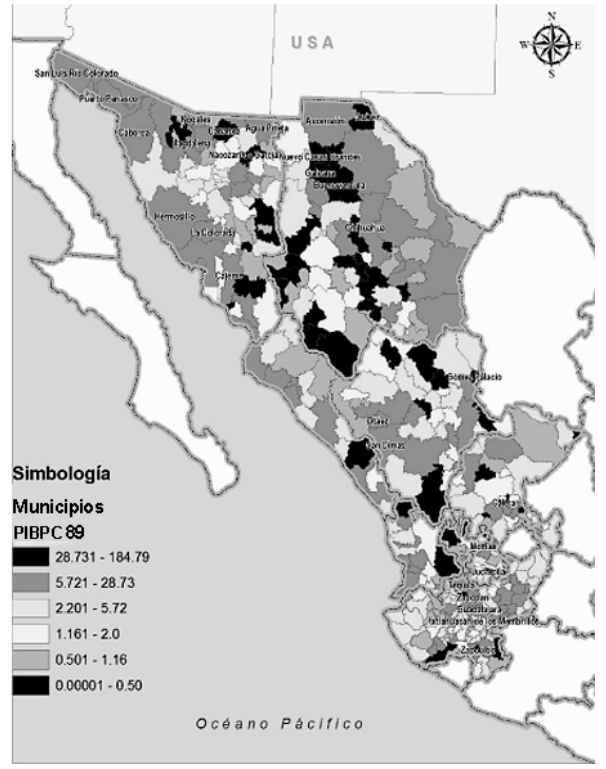

Fuente: Elaboración propia con datos de INEGI, Censos Económicos 2004 y producto interno bruto por entidad federativa, México.

Los resultados del modelo de regresión realizado mediante el procedimiento del método de mínimos cuadrados no lineales, identificado con la ecuación 2.5 , se reportan en el cuadro 1 , las convergencias $\sigma$ y $\beta$, para el caso de los municipios de los estados contiguos a Nayarit y Sinaloa, a la región de Sinaloa y Nayarit, así como los correspondientes a los municipios de Durango, Jalisco, Zacatecas, Sonora y Chihuahua.

El modelo econométrico es de corte transversal para un periodo de 17 ańos, comprendidos entre 1989 y 2006, época en que México transita por una etapa de apertura comercial de su economía. En el modelo se utilizó como variable dependiente la tasa de crecimiento promedio anual del periodo señalado y como independiente el logaritmo del producto per cápita del año 1989, relación que en general se utiliza en estos modelos.

En la primera columna se presenta la región o los estados, de los cuales se consideraron los municipios. En las columnas 2 y 3 se reporta la convergencia $\sigma$ obtenida del ingreso per cápita de los dos años considerados para el estudio. En la columna siguiente se presenta el resultado del parámetro de convergencia y su estadístico asociado en la columna siguiente. Para luego seguir con el indicador de la velocidad de convergencia, a continuación el coeficiente de determinación $\left(\mathrm{r}^{2}\right)$, y finalmente se 


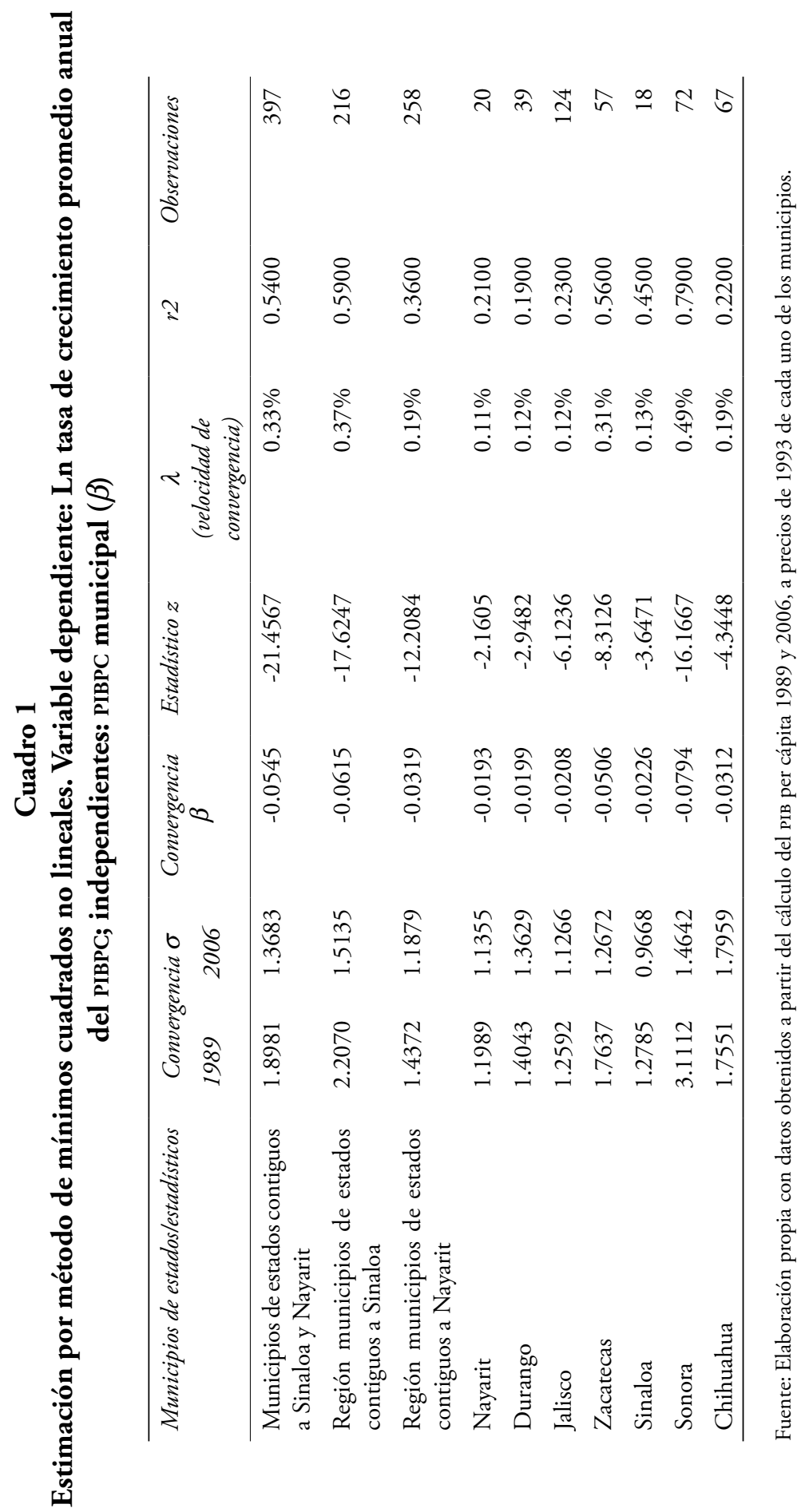


consigna el número de municipios que se tomaron como observaciones utilizadas en el modelo.

Los resultados de computar la desviación estándar en el año 1989 fue de 1.89 y disminuyó a 1.36 en 2006 para los municipios de estados contiguos a Sinaloa y Nayarit. Los municipios de los estados considerados en el estudio logran convergencia, salvo los de Chihuahua que reportan leve divergencia $\sigma$, pues en el primer año es de 1.75 y en el segundo de 1.79.

Por otra parte, de acuerdo con la literatura, para identificar la relación de la convergencia $\beta$ se espera que el signo de este parámetro sea negativo, relación que se obtuvo en todos los modelos, además el estadístico con valor absoluto mayor a 2 , da cuenta de que es estadísticamente distinto de cero a $5 \%$ de nivel de significancia, tendencia que se ilustra mediante gráficas.

El parámetro de lambda $(\lambda)$ que representa la tasa de convergencia hacia el supuesto estado estacionario, es variable de acuerdo con los municipios de los estados tomados en el estudio. Considerada como una región compuesta por los municipios de Nayarit, Durango, Jalisco, Zacatecas, Sinaloa, Sonora y Chihuahua reportan un guarismo de $0.33 \%$, que es superior al que informan individualmente los estados, salvo Sonora; por ese parámetro se tiene conocimiento que la brecha del ingreso entre los municipios tiende a disminuir a una tasa aproximada de $0.33 \%$ por año. Cabe señalar que en otros estudios, en que se asocian países o regiones de los países se ha encontrado una tasa de $2 \%$, porcentaje que en algunos casos se presenta como ley de hierro de la velocidad de convergencia.

Ahora bien, considerando las regiones de Sinaloa y de Nayarit, se observan resultados que contrastan, por una parte la velocidad de convergencia de la primera región se ubica en $0.37 \%$ y la de Nayarit en $0.19 \%$, lo cual indica que la desigualdad de los municipios de la región de Sinaloa podrían abatir la desigualdad en $50 \%$ del tiempo que se requeriría para abatirla en Nayarit. Esta aseveración la refuerza el coeficiente de determinación, que para el caso de la región de Sinaloa la variable dependiente explica en $60 \%$ el comportamiento; pero para Nayarit sólo 36\%, no obstante que para la primera región se consideraron sólo 216 municipios y para Nayarit 258 (gráfica I a-c).

Por otra parte, en el ámbito municipal, si bien todos reportan convergencia $\beta$ negativa que por ser precedida de convergencia $\sigma$ decreciente en los dos periodos de análisis, se puede afirmar que existe convergencia absoluta y se ilustra en las gráficas de cada uno de los estados; la velocidad para abatir el rezago es variada, mientras en Nayarit se abatiría a una tasa de $0.11 \%$ anual y la variable utilizada explica en $21 \%$ a las dependientes, el estado de Sonora reporta una tasa de $0.50 \%$, además por el coeficiente de determinación la variable utilizada explica en casi $80 \%$ el modelo. También se presenta el mapa II (véase cuadro 1; gráfica II a-f). 


\section{Conclusiones}

En términos generales se acepta al PIBPC como una medida de bienestar social de la población, incluso en los Objetivos del Milenio, acordados por las Naciones Unidas, se tomó el PIBPC como un indicador de la situación de la población. Ese indicador es fundamental para analizar la convergencia económica. En principio, por los resultados de $\sigma$-convergencia, que fue decreciente de 1989 a 2006, se evidencia la convergencia sigma y por la relación que reporta convergencia beta, es probable la tendencia de convergencia en los municipios analizados.

La investigación muestra que la convergencia $\sigma$ en los municipios contiguos a Nayarit (Durango, Jalisco, Sinaloa y Zacatecas) es de menor escala que la convergencia que se presenta en los municipios contiguos a Sinaloa (Nayarit, Sonora, Durango y Chihuahua). Sin embargo, por el $\lambda$ obtenido para la región, se puede aceptar la aseveración de la hipótesis de convergencia que enuncia que los municipios relativamente atrasados tienden a acercarse a los municipios relativamente prósperos a una tasa muy lenta. Se observa que los municipios contiguos a Nayarit tienden a converger alrededor de dos veces más rápido que los contiguos a Sinaloa.

En consecuencia, se reconoce que México cuenta con políticas sociales y sectoriales con marcadas dimensiones regionales, pero carece, en la actualidad, de una política regional como tal, a pesar de que se describen estrategias y están presentes en los programas de reforma económica, privatización, descentralización y política social (Chapa, 2000: 212). Sin embargo, la apertura comercial ha dibujado en México cierta disparidad regional, donde el norte se presenta relativamente desarrollado, la región central con una situación tradicional mejor, pero en la parte sur, con un marcado atraso económico. Además, al parecer al interior de cada una de ellas se presentan diferencias marcadas de desigualdad, a las que habría que sumarle las discrepancias dentro de los estados, concretamente en el ámbito de los municipios.

En este trabajo, si bien se fundamentó la convergencia en el ámbito municipal, se observa que en cada uno de los estados una parte de la población reporta ingresos económicos bajos, dicha población tiende a incrementarse, y por la velocidad de convergencia, de continuar esta tendencia se puede decir que deberán transcurrir muchos años para que la población con bajos ingresos alcance a los que tienen mejores ingresos. Faltaría precisar la cantidad de población de los municipios de esa región que ha visto disminuido sus ingresos y la relación de la población con otros indicadores socioeconómicos. 


\section{Gráfica I}

Relación entre la tasa de crecimiento del PIBPC durante 17 años y el periodo inicial

I a. Región de municipios de estados contiguos a Nayarit y Sinaloa

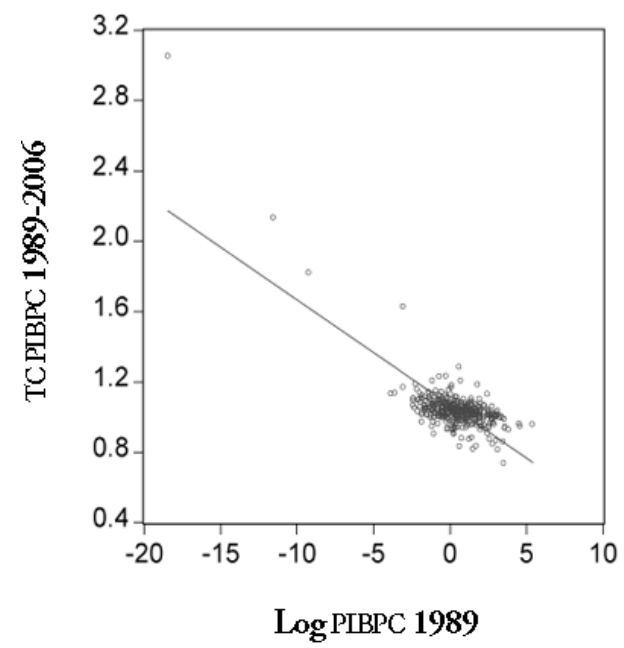

Fuente: Elaboración propia con datos obtenidos a partir del cálculo del PIB per cápita 1989 y 2006, a precios de 1993 de cada uno de los municipios (para las gráficas a-c).

I b. Región de municipios de estados colindantes con Nayarit

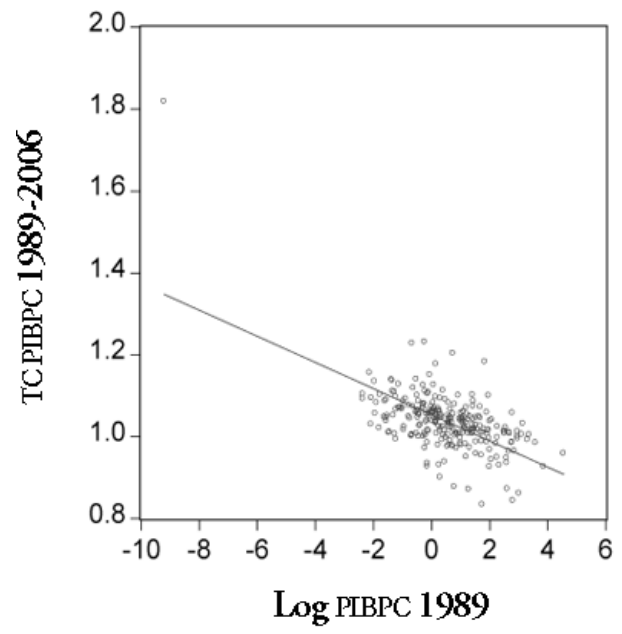


I c. Región de municipios de estados colindantes con Sinaloa

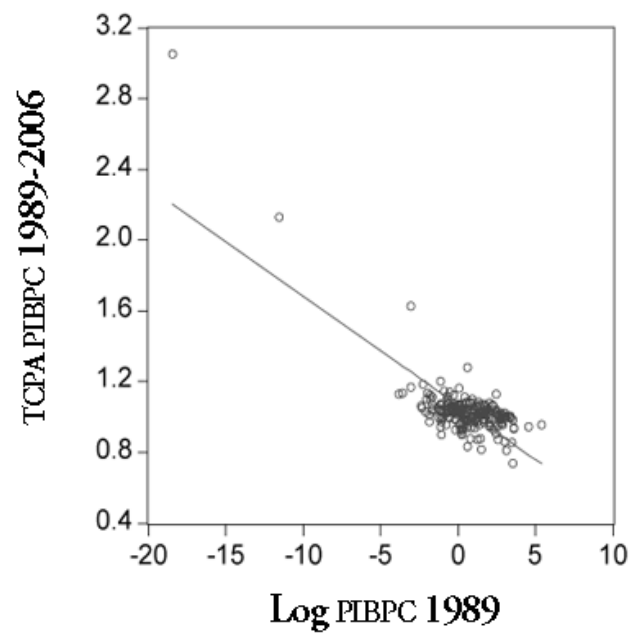

Gráfica II

Relación entre la tasa de crecimiento y el producto inicial en los municipios de los estados referidos

II a. Municipios de Nayarit

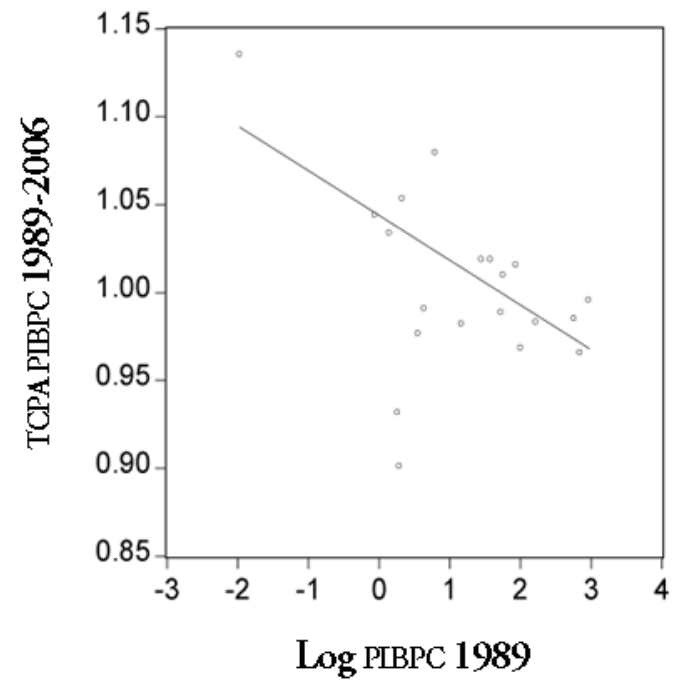

Fuente: Elaboración propia con datos obtenidos a partir del cálculo del PIB per cápita de 1989 y 2006, a precios de 1993 para cada uno de los municipios (para las gráficas a-f). 
II b. Municipios de Durango

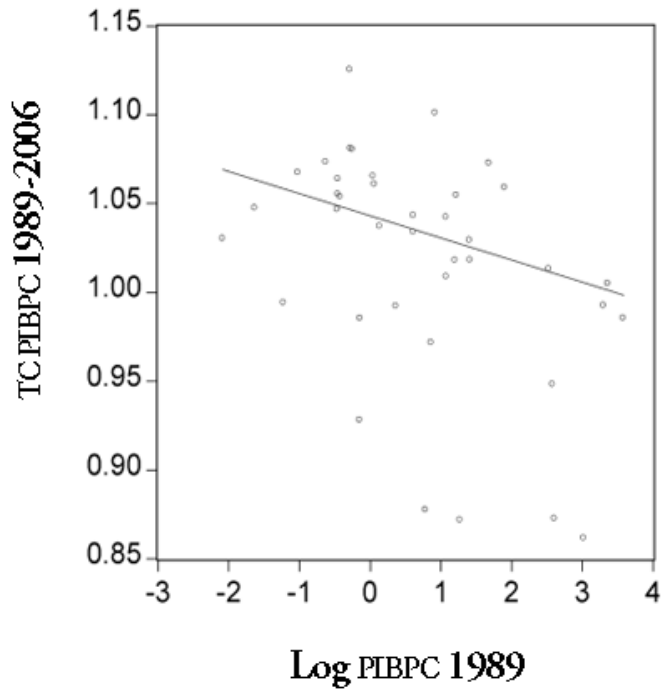

II c. Municipios de Jalisco

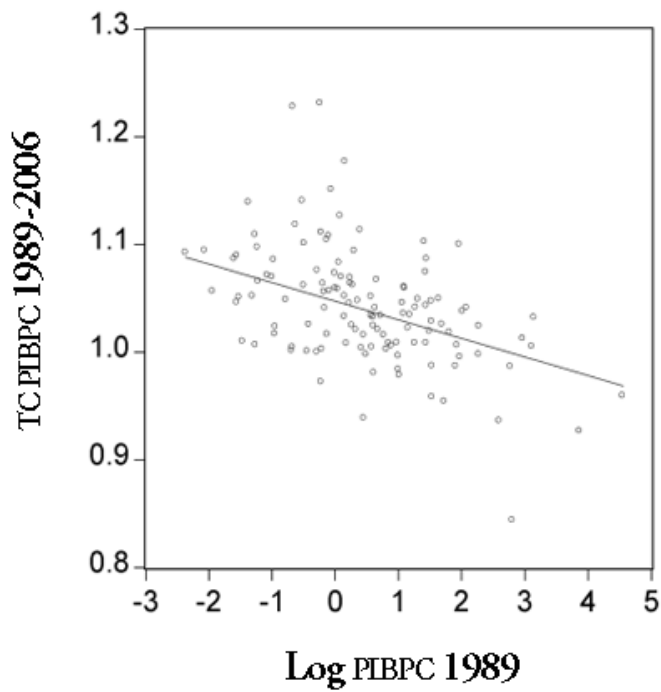


II d. Municipios de Zacatecas

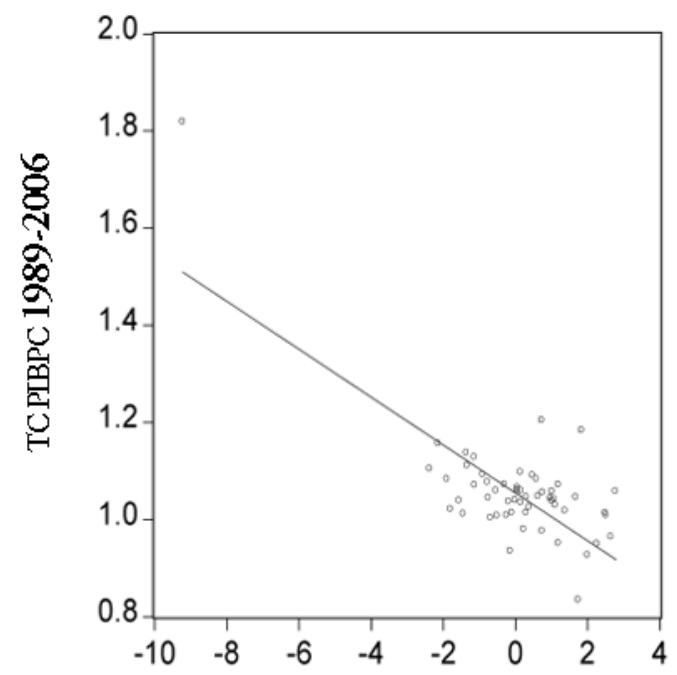

$\log$ PIBPC 1989

II e. Municipios de Sinaloa

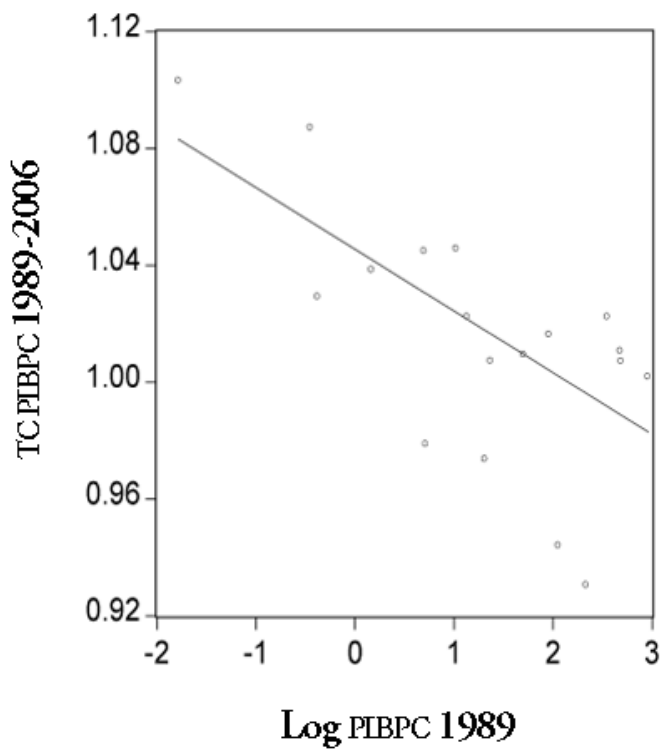


II f. Municipios de Chihuahua

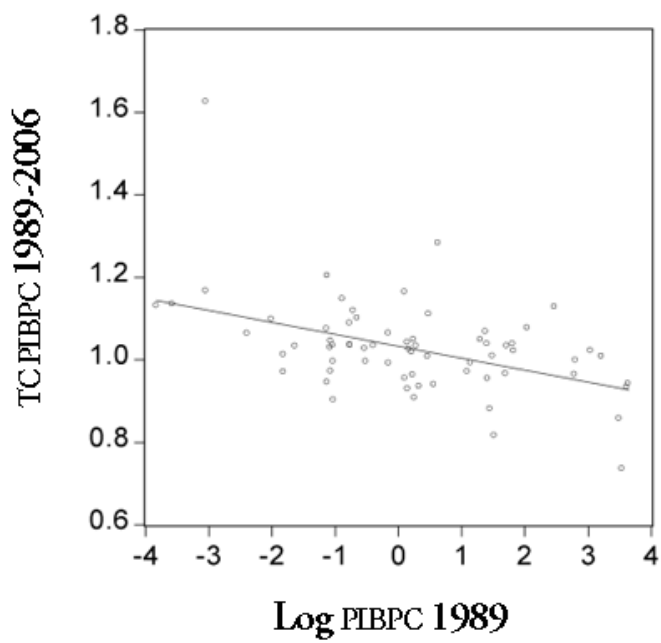




\section{Bibliografía}

Aguado-Moralejo, Itziar, Miguel Echebarria, José María Carmen y Barrutia Legarreta (2009), "El desarrollo sostenible a lo largo de la historia del pensamiento económico", Revista de Economía Mundial, <http://redalyc.uaemex.mx/src/inicio/ArtPdfRed.jsp?iCve= 86611886004>, 12 de septiembre de 2010.

Aroca, Patricio y Mariano Bosch (2000), "Crecimiento, convergencia y espacio en las regiones chilenas: 1960-1998”, Estudios de Economía, <http://redalyc.uaemex.mx/redalyc/src/inicio/ArtPdfRed. jsp?iCve=22127202>, 12 de septiembre de 2010.

Asuad, Normand y Luis Quintana (2006), “Convergencia espacial en el crecimiento económico de las entidades federativas de México, 1940-2004", en xvi Colegio Mexicano de Economía Matemática y Estadística, Universidad Veracruzana, México, pp.1-30.

Barceinas, Fernando y José Luis Raymond (2005), "Convergencia regional y capital humano en México, de los años 80 al 2002”, Estudios Económicos, <http://redalyc.uaemex.mx/src/inicio/ArtPdfRed. jsp?iCve=59713036005>, 12 de septiembre de 2010 .

Chapa, J. Benjamín (2000), "Desarrollo regional y política estructural en México”, Espiral, 6 (17), Universidad de Guadalajara, Guadalajara, pp. 211-221.

Corona-Jiménez, Miguel Ángel (2003), "Efectos de la globalización en la distribución espacial de las actividades económicas”, Comercio Exterior, 53 (1), Bancomext, México, pp. 48-56.

Díaz-Bautista, Alejandro y Manuel Díaz (2003), "Capital humano y crecimiento económico en México", Comercio Exterior, 53 (11), Bancomext, México, pp. 1012-1023.

Esquivel, Gerardo (1999), "Convergencia regional en México, 19401995”, El Trimestre Económico, 66 (4), Fondo de Cultura Económica, México, pp. 725-761.

Germán-Soto, Vicente (2005), "Generación del producto interno bruto mexicano por entidad federativa, 1940-2002”, El Trimestre Eco- 
nómico, 3 (287), Fondo de Cultura Económica, México, pp. 617-653.

Gujarati, Damodar (2005), Econometría, Mc Graw Hill, México.

INEGI (Instituto Nacional de Estadística, Geográfica e Informática), Censos y Conteos Generales de Población 1980, 1990, 1995, 2000 y 2005, INEGI, México, www.inegi.gob.mx.

Krugman, Paul (1991) "Increasing Returns and Economic Geography", Journal of Political Economy, vol. 99 (3), pp. 483-499, <http:// pioneer.netserv.chula.ac.th/-kkornkar/inter\%20trade\%20course/ geography.pdf>, 12 de septiembre de 2010.

Martínez-Coll, Juan Carlos (2001), "El crecimiento económico" en la economía de mercado, virtudes e inconvenientes, <http://www. eumed.net/cuursecon/ 18/index.htm>, 3 de febrero de 2008.

Meier, Gerald M. y Dudley Seers, (1986), Pioneros del desarrollo, Tecnos, Madrid.

Messmacher, Miguel (2000), "Desigualdad regional en México. El efecto del TLCAN y otras reformas estructurales", Documento de Investigación 2000-4, División de Investigación Económica, Banco de México.

Ocegueda-Hernández, Juan Manuel (2007), "Apertura comercial y crecimiento económico en las regiones de México", Investigación Económica, LXVI (262), Universidad Nacional Autónoma de México, México, pp. 89-137.

Quah, Danny (1999), "Ideas determining convergence clubs", Working Paper, Economic Department, London School of Economics, Londres.

Rodríguez-Orregia, Eduardo (2005), "Regional disparities and determinants of growth in Mexico", Annals of Regional Science, 39, Universidad Iberoamericana, México, D.F., pp. 207-220, DOI: 10.1007/s00168-004-0218-5. 
Ros-Bosch, Jaime y Peter Skott (1995), "Dinamic Effects of Trade Liberalization and Currency Overvaluation under Conditions of Increasing Returns", The Manchester School, Manchester.

Sala-i-Martin, Xavier (1994), Apuntes de crecimiento económico, Antoni Bosh Editor, Barcelona.

Solow, Robert (1956), "A Contribution to the Theory of Economic Growth", Quarterly Journal of Economics, 70 (1), The міт Press, pp. 65-94.

Valdivia-López, Marcos (2007), "Análisis de convergencia espacial en México a partir de remesas internacionales e inversión extranjera directa”, documento de trabajo, Programa de Estudios Regionales, CRIM-unAm, México.

Villaverde, José y Blanca Sánchez-Robles (1998), "Disparidades provinciales y clubes de convergencia en España”, Estudios Regionales, 3 (52), Universidades Públicas de Andalucía, Sevilla, pp. 177-199.

Recibido: 3 de noviembre de 2009. Aceptado: 26 de febrero de 2010.

Eduardo Meza-Ramos. Es doctor por la Universidad Autónoma de Baja California, México; es maestro y licenciado por la Universidad Autónoma de Nayarit. Actualmente es investigador nivel I en el Sistema Nacional de Investigador (SNI) y es coordinador de la maestría en Desarrollo Económico Local, que se imparte en la Unidad Académica de Economía de la UAN. Su línea de investigación es: desarrollo local y migración. Entre sus publicaciones destaca: Estructura económica y migración interna en Nayarit. Un análisis econométrico, UAN, México (2009).

Zulema Berenice Naya-Flores. Es estudiante del programa de licenciatura en Comercio Internacional de la Universidad Autónoma de Sinaloa. Realizó una estancia de investigación, durante el XIII Verano de Investigación Científica del Pacífico, del programa Delfin, en la UAN. 